\title{
Development of the Japanese Medication Adherence Scale and Verification of Its Reliability and Validity in Hypertensive Patients
}

\author{
Rika Shimada1, Yasuaki Dohi' ${ }^{2}$, Kazunori Kimura ${ }^{3}$, Satoshi Fujii ${ }^{4}$ \\ ${ }^{1}$ Department of Critical Care Nursing, Graduate School of Nursing, Nagoya City University, Nagoya, Japan \\ ${ }^{2}$ Department of Cardio-Renal Medicine and Hypertension, Graduate School of Medicine, Nagoya City University, \\ Nagoya, Japan \\ ${ }^{3}$ Pharmaceutical Department, Nagoya City University Hospital, Nagoya, Japan \\ ${ }^{4}$ Department of Laboratory Medicine, Asahikawa Medical University, Asahikawa, Japan \\ Email: shimada@med.nagoya-cu.ac.jp
}

Received 13 January 2015; accepted 3 March 2015; published 9 March 2015

Copyright (C) 2015 by authors and Scientific Research Publishing Inc.

This work is licensed under the Creative Commons Attribution International License (CC BY).

http://creativecommons.org/licenses/by/4.0/

(c) () D Den Access

\section{Abstract}

A 32-Item Japanese Medication Adherence Scale had been developed as a tool for evaluating the medication-taking behavior of hypertensive patients and predicting therapeutic efficacy, and an Internet survey of 990 hypertensive patients throughout Japan was performed. As a result, factor 1 "Expectation of pharmacological efficacy" ( 9 items), factor 2 "Motivation to be self-controlled in taking medication" (6 items), and factor 3 "Negative feelings about taking medication" (4 items) were identified, comprising a total of 19 items. The scale was highly reliable. Because it proved capable of discriminating between the 2 groups with different medication-taking status and blood pressure, its validity had also been confirmed.

\section{Keywords}

Drug Therapy, Hypertension, Measure, Medication Adherence Scale

\section{Introduction}

Hypertension is a major risk factor for disorders such as coronary artery disease, heart failure, cerebral stroke, and renal failure, and it is important to keep high blood pressure under control. Essential hypertension, which 
accounts for $90 \%$ of cases of hypertension, may be caused by factors including obesity, stress, smoking, alcohol, and excessive salt intake, in addition to genetic factors [1]. It is standard medical practice to provide health education on these factors, as well as drug treatment. However, these factors are heavily dependent on patient selfcare, and many hypertensive patients find it difficult to control their own blood pressure [2].

It is estimated that approximately 20 million men and approximately 17 million women in Japan suffer from hypertension. The increase in hypertensive patients has also influenced increase of medical expenses. The medical expenses used for the hypertensive disease in 2004 were 1900 billion yen [3]. If inadequately controlled blood pressure is left untreated and potential future secondary diseases are not prevented, this will be detrimental not only to individuals but also for society at large.

Recently, the concept of adherence has come into use as an outcome related to medication-taking behavior in an attempt to describe patient behavior in a holistic manner [4]. Adherence refers to whether patients are capable of implementing treatment with medication correctly. It is affected by a range of different factors, including the patient's own values regarding health, their capacity to understand the disease and its treatment, their attitude to medication and ability to take medication, and the state of control of their disease [5]. It is suggested that reducing the number of tablets and the frequency of medications are effective in improving adherence [6] [7]. Research on medication adherence is still in its infancy. In Japan, a scale has yet to be developed for the appropriate measurement of medication-taking behavior in hypertensive patients, while the factors associated with medication adherence have not yet been elucidated. Thus, the aim of this study was to develop a scale to evaluate adherence among hypertensive patients and confirm the validity and reliability of the new measure.

\section{Methods}

\subsection{Development of the Medication Adherence Measure: Interview to Clarify Factors}

After obtaining approval from the ethics committee of the Nagoya City University, patients aged $\geq 35$ and $<80$ years who were attending the clinic of the Department of Cardiovascular Medicine as outpatients, were taking antihypertensive medication, and had consented to participate in this study were invited. Patients with serious liver or kidney damage, uncontrolled diabetes, or endocrine disorders were excluded. Semi-structured interview was undertaken to each participant. The contents of the interviews were capacity to take medication, awareness of medication, what sort of information was given, and his or her behavior toward to taking medication. The 59 subjects included 26 men and 33 women with a mean age of $68.1 \pm 9.9$ years. Blood pressure at the first survey was classified according to the Guidelines for the Management of Hypertension (Japanese Society of Hypertension), with 22 patients (37.3\%) having normal blood pressure, 20 (33.9\%) high normal blood pressure, 15 (25.4\%) grade 1 hypertension, 1 (1.7\%) grade 2 hypertension and 1 (1.7\%) grade 3 hypertension. Six factors associated with medication-taking behavior were isolated by analysis of the results of the interviews: "Trust in doctors and medication," "Interest in pharmacological action," "Desire to acquire knowledge about medication," "Making an effort to take medication properly," "Values regarding health," and "Thoughts on prescribed medication.”

\subsection{Preparation of the Scale}

\subsubsection{The Japanese Medication Adherence Measure Version 1}

Sixty questions were formulated with reference to the categories formed by these 6 factors, and the Japanese Medication Adherence Measure version 1 was prepared. Responses were requested on a 5-point scale from "Very applicable" to "Not applicable at all." A survey was carried out by an internet research company. All subjects were Japanese who were taking antihypertensive medication daily. The results of a survey of 441 male and female patients aged 50 - 69 years taking antihypertensive medication (271 men, 170 women, mean age $59.4 \pm 5.3$ years) carried out using this measure were then verified.

\subsubsection{The Japanese Medication Adherence Measure Version 1}

Based on the Japanese Medication Adherence Measure version 1, the Japanese Medication Adherence Measure version 2, which consisted of 4 factors comprising a total of 32 items scored on a 5-point scale from "Very applicable" to "Not applicable at all," was prepared. It was then used to carry out an Internet survey entitled "Questionnaire about how you take your medication" of 990 men and women from throughout Japan who were 
taking medication to treat hypertension. Mean age was $59.5 \pm 10.7$ (40 - 79) (Table 1).

\subsection{Statistical Analysis}

Reliability was investigated in terms of internal consistency and the test-retest method, with retesting performed 2 weeks after the first survey. Discriminant validity was investigated by dividing patients into groups with blood pressure and number of times they forgot to take their medication as independent variables, and using the t-test to investigate the differences between the groups with subscale scores as dependent variables. The level of statistical significance was set at $5 \%$.

\section{Results}

\subsection{Verification of Results of a Survey Using the Japanese Medication Adherence Measure Version 1}

The sum total of distribution reached to $45 \%$ by 4 th factor. The scree plots of principal component analysis and a comparison of the factor content of each factor solution also suggested that a 4-factor solution was appropriate. Then, factor analysis was performed for a 4-factor solution using the principal factor method and promax rotation. Items for which factor loading were $<0.40$ and those for which loading was similar for multiple factors were excluded. Then, factor analysis was repeated and it was determined that 32 items were appropriate. These 32 items were categorized as follows.

Factor 1, "Motivation to be self-controlled in taking medication," comprised 12 items including "I sometimes can't take my medication when I'm tired" and "It is difficult to take my medication because of its size or shape."

Factor 2, "Expectation of pharmacological efficacy," comprised 10 items including "I believe medication is effective" and "I take it so my condition won't get worse."

Factor 3, "Concern about side effects," comprised 8 items including "I am worried about the possible side effects of medication" and "I would like to know how strong the medication I am taking is."

Table 1. Sample character.

\begin{tabular}{|c|c|c|}
\hline Characteristics & & Number (\%) \\
\hline \multirow{2}{*}{ Sex } & Male & $562(56.8)$ \\
\hline & Female & $428(43.2)$ \\
\hline \multirow{7}{*}{ Frequency of checking blood pressure per day } & Never & $206(20.8)$ \\
\hline & Once & 383 (38.7) \\
\hline & 2 times & $270(27.3)$ \\
\hline & 3 times & $40(4.0)$ \\
\hline & 4 times & $7(0.7)$ \\
\hline & More than 5 times & $8(0.8)$ \\
\hline & No answer & $76(7.7)$ \\
\hline \multirow{6}{*}{ Classification of blood pressure } & Optimum & $58(5.9)$ \\
\hline & Normal & $232(23.4)$ \\
\hline & High normal & 374 (37.8) \\
\hline & Grade 1 & 269 (27.2) \\
\hline & Grade 2 & $53(5.4)$ \\
\hline & Grade 3 & $4(0.4)$ \\
\hline \multirow{5}{*}{ Frequency of taking medicine per day } & Once & 445 (44.9) \\
\hline & 2 times & 315 (31.8) \\
\hline & 3 times & $202(20.4)$ \\
\hline & 4 times & $21(2.1)$ \\
\hline & More than 5 times & $7(0.7)$ \\
\hline \multirow{5}{*}{ Frequency of forgot to take medicine per week } & None & 755 (76.3) \\
\hline & Once & $170(17.2)$ \\
\hline & 2 times & 45 (4.5) \\
\hline & 3 times & $10(1.0)$ \\
\hline & More than 4 times & $10(1.0)$ \\
\hline
\end{tabular}

Blood pressure was classified according to the guidelines for the management of hypertension (Japanese Society of Hypertension, 2014). 
Factor 4, "Dealing with forgetting to take medication," comprised 2 items, "I do not think forgetting to take medication once or twice is a problem" and "I do not think forgetting to take medication can be helped."

Cronbach's $\alpha$ coefficient was calculated for each factor to confirm internal consistency, and it was 0.856 for factor 1, 0.810 for factor 2, 0.777 for factor 3, and 0.679 for factor 4 .

\subsection{Verification of the Reliability and Validity of the Japanese Medication Adherence Measure Version 2}

Four of the 32 items, for which the ceiling effect was evident, had been excluded. The remaining 28 items were analyzed. From the results of scree plots of principal component analysis of scores and a comparison of the factor content of each factor solution, it was determined that a 3-factor solution was appropriate, and factor analysis was performed for a 3-factor solution using the principal factor method and promax rotation. Items for which factor loading were $<0.40$ and those for which loading was similar for multiple factors were excluded. Then, factor analysis was repeated. It was determined that 19 items were appropriate, and these were categorized as follows (Table 2).

Factor 1, "Expectation of pharmacological efficacy," comprised 9 items including "I believe medication is effective" and "I think medication is important."

Factor 2, "Motivation to be self-controlled in taking medication," comprised 6 items including "I make my own decision as to whether or not to take my medication" and "I choose for myself which medication I want to take.”

Factor 3, "Negative feelings about taking medication" comprised 4 items, including "I want to stop taking medication" and "I am worried about the possible side effects of medication."

Cronbach's $\alpha$ coefficient was calculated for each factor to confirm internal consistency. Cronbach's $\alpha$ coefficient was 0.829 for factor $1,0.743$ for factor 2 , and 0.616 for factor 3 . The correlation coefficients for subscale scores measured during the first survey and by the retest method 2 weeks later (Table 3). The correlation coefficients between factors were (Table 4). The patients were divided according to self-measured blood pressure following the Guidelines for the Management of Hypertension (Japanese Society of Hypertension, 2009) into those with optimal or normal blood pressure and those with high normal blood pressure or hypertension. The statistical difference of 2 groups were determined by the t-test, scores for all subscales and total scores were significantly higher in the normal blood pressure group $(\mathrm{p}<0.05)$ (Table 5). Then, they were divided into those

Table 2. Factor loading of adherence scale among hypertensive patients.

\begin{tabular}{|c|c|c|c|}
\hline \multirow{2}{*}{ Items } & \multicolumn{3}{|c|}{ Factor loading } \\
\hline & F1 & F2 & F3 \\
\hline \multicolumn{4}{|l|}{ Factor 1: Expectation of pharmacological efficacy $(\alpha=0.829)$} \\
\hline I believe medication is effective. & 0.675 & 0.067 & -0.102 \\
\hline I trust the doctor who prescribes my medication. & 0.662 & 0.027 & -0.158 \\
\hline I am serious about taking medication. & 0.644 & -0.085 & 0.035 \\
\hline I think medication is important. & 0.642 & 0.045 & 0.025 \\
\hline I take it so my condition won't get worse. & 0.606 & -0.068 & 0.171 \\
\hline I expect the medication to have an effect. & 0.590 & -0.028 & 0.187 \\
\hline I always try to take it when the time comes round. & 0.577 & 0.249 & 0.010 \\
\hline I take it in the way the doctor told me to. & 0.546 & -0.153 & 0.009 \\
\hline I am accustomed to taking medication. & 0.535 & 0.034 & 0.031 \\
\hline \multicolumn{4}{|l|}{ Factor 2: Motivation to be self-controlled in taking medication ( $\alpha=0.743)$} \\
\hline I make my own decision as to whether or not to take my medication. & 0.180 & 0.675 & -0.116 \\
\hline I choose for myself which medication I want to take. & 0.217 & 0.671 & -0.131 \\
\hline Even if I ask medical professionals a question about medication, they won't answer me. & -0.118 & 0.528 & 0.128 \\
\hline I don’t know why I'm taking medication. & -0.220 & 0.523 & 0.079 \\
\hline Even if you are unable to take medication properly, that's all right. & -0.174 & 0.467 & 0.044 \\
\hline I'm taking medication even though I don't know what effect it has. & -0.129 & 0.442 & 0.071 \\
\hline \multicolumn{4}{|l|}{ Factor 3: Negative feelings about taking medication $(\alpha=\mathbf{0 . 6 8 9})$} \\
\hline I want to stop taking medication. & -0.125 & -0.067 & 0.660 \\
\hline If possible, I would prefer not to take medication. & 0.087 & -0.188 & 0.607 \\
\hline I am worried about the possible side effects of medication. & 0.027 & 0.248 & 0.457 \\
\hline I am concerned about the efficacy of medication. & 0.290 & 0.084 & 0.434 \\
\hline
\end{tabular}


Table 3. Correlation between test and retest of the medication adherence scale.

\begin{tabular}{|c|c|c|c|c|}
\hline & \multicolumn{4}{|l|}{ Retest } \\
\hline & & Factor 1 & Factor 2 & Factor 3 \\
\hline \multirow{3}{*}{ Test } & Factor 1: Expectation of pharmacological efficacy & $0.697^{* *}$ & $-0.400^{* *}$ & 0.046 \\
\hline & Factor 2: Motivation to be self-controlled in taking medication & $-0.408^{* *}$ & $0.663^{* *}$ & $0.155^{* *}$ \\
\hline & Factor 3: Negative feelings about taking medication More table copy ${ }^{\mathrm{a}}$ & 0.021 & $0.159^{* *}$ & $0.689^{* *}$ \\
\hline
\end{tabular}

${ }^{* *} \mathrm{p}<0.01$

Table 4. Correlation between the domains of the medication adherence scale.

\begin{tabular}{|c|c|c|c|}
\hline Factor & Factor 1 & Factor 2 & Factor 3 \\
\hline Factor 1: Expectation of pharmacological efficacy & 1.000 & $-0.370^{* *}$ & $0.126^{* *}$ \\
\hline Factor 2: Motivation to be self-controlled in taking medication & - & 1.000 & $0.242^{* *}$ \\
\hline Factor 3: Negative feelings about taking medication copy & - & - & 1.000 \\
\hline
\end{tabular}
$\stackrel{* *}{\mathrm{p}}<0.01$.

Table 5. Sample t-test results from comparing variables according to blood pressure.

\begin{tabular}{cccccc}
\multirow{2}{*}{ Total score } & \multicolumn{2}{c}{ Normal $(\mathrm{n}=290)$} & \multicolumn{2}{c}{ High $(\mathrm{n}=700)$} & Significance \\
\cline { 2 - 5 } & Average & SD & Average & SD & 0.036 \\
Factor 1 & 18.0 & 4.7 & 18.7 & 4.7 & 0.000 \\
Factor 2 & 24.2 & 3.3 & 23.1 & 3.6 & 0.002 \\
Factor 3 & 11.1 & 2.8 & 10.5 & 2.9 & 0.017 \\
\hline All & 53.3 & 5.6 & 52.3 & 6.0 & 0.0 \\
\hline
\end{tabular}

who had forgotten to take their medication at least once during the previous week and those who had not forgotten to take it. The 2 groups were compared using the t-test. All subscale scores were significantly higher for those who had not forgotten to take their medication $(\mathrm{p}<0.01)$ (Table 6).

\section{Discussion}

The word "adhere" means "stick to" or "hold fast" [8]. The concept of "adherence" has become more widely accepted worldwide. "Adherence" is a patient-centered term that refers to patients actively participating in medical treatment of their own accord and sticking to the treatment method. Patients with high adherence can thus be expected to participate in the treatment of their own accord in the endeavor to increase its therapeutic efficacy. Adherence is affected by numerous factors, including the type of treatment, patient factors, and factors related to medical professionals. A prominent feature of this concept is that adherence is understood in terms of the mutual relationship between patients and medical professionals.

In the treatment of cardiovascular diseases, factors that affect adherence can be broadly divided into those concerning patients themselves and those concerning their relationships with other people [5]. Factors concerning patients themselves include the state of their disease, health risks (smoking, obesity, lack of exercise, blood lipids, diabetes, etc.), outlook on health and view of cardiovascular disease, the health education they have received, what targets have been set, motivation, desire for change, and belief in their own ability to achieve results [9]. Factors concerning their relationships with other people include patients' social backgrounds, whether or not they have a support framework and their relationships with medical professionals [10] [11]. These factors interact with each other to regulate patients' adherence.

Within the conceptual structure determining medication adherence by hypertensive patients in our study, "Expectation of pharmacological efficacy" and "Motivation to be self-controlled in taking medication" improve adherence, whereas "Negative feelings about taking medication" reduce it.

The state of taking medicine by hypertensive patients is also affected by adherence. In addition to generally identified factors, the method of administration of medication also contributes to adherence of hypertensive pa- 
Table 6. Sample t-test results from comparing variables according to forget medication.

\begin{tabular}{cccccc}
\hline \multirow{2}{*}{ Total score } & \multicolumn{2}{c}{ Normal $(\mathrm{n}=290)$} & \multicolumn{2}{c}{ High $(\mathrm{n}=700)$} & Significance \\
\cline { 2 - 5 } & Average & SD & Average & SD & 0.000 \\
Factor 1 & 18.1 & 4.6 & 19.8 & 4.6 & 0.003 \\
Factor 2 & 23.6 & 3.5 & 22.8 & 3.6 & 0.009 \\
Factor 3 & 10.8 & 2.9 & 10.2 & 2.7 & 0.509 \\
All & 52.5 & 5.8 & 52.8 & 6.1 & 0.9 \\
\hline
\end{tabular}

tients. Compound formulations, which combine several active ingredients in a single tablet, have been reported to have an increased rate of use compared with multidrug therapy in which several medications are taken at the same time [6]. Reducing the number of medications taken at once [12], the number of medications taken per day, and the number of doses per day has also been shown to be effective in improving adherence [7], suggesting that simpler methods of taking medication result in higher adherence. It has also been pointed out that providing sufficient information increases adherence and improves medication-taking behavior, resulting in improved blood pressure control [13]. We found that the expectation of pharmacological efficacy is an expression of the desire to keep blood pressure under good control, while the motivation to be self-controlled on the basis of an understanding of the medication involved overrides negative feelings about taking medication to improve adherence.

From previous studies, factors affecting adherence have been shown to include patient's socioeconomic status [14], patient knowledge, method of administration, anxiety about side effects, and the medical professional-patient relationship [6] [7] [9]-[12] [15]. In addition to practical patient intervention strategies, it will be necessary to obtain evidence on the outcomes of interventions in Japan. Scales for evaluating adherence by hypertensive patients have been developed overseas, including Self-reported Medication-taking Scaleby Morisky [16] from the 1980s and the 8-Item Medication Adherence Measure [17]. However, few studies using these scales have been carried out in Japan, and the reliability and validity of the Japanese versions have yet to be adequately verified.

The Japanese Medication Adherence Measure version 2 is the first adherence scale that demonstrates its reliability and validity. Reliability was investigated in terms of internal consistency and the retest method. Discriminated validity was investigated by dividing patients into groups with blood pressure and number of times they forgot to take their medication as independent variables, and using the t-test to investigate the differences between the groups with subscale scores as dependent variables. The Japanese Medication Adherence Measure version 2 proved to be a reliable, simple and valid method for assessing patients' medication-taking behavior in clinical practice. It will be a valuable tool for predicting therapeutic efficacy.

\section{Conclusion}

A 32-Item Japanese Medication Adherence Scale has been developed and evaluated. Identified factor contains 19 items, factor 1, "Expectation of pharmacological efficacy", factor 2, "Motivation to be self-controlled in taking medication", and factor 3, "Negative feelings about taking medication". The measure was highly reliable, and because it proved capable of discriminating between 2 groups with different medication-taking status and blood pressure, its validity has also been confirmed. This would enable the widespread assessment of medication-taking behavior in clinical practice in Japan and provide a valuable tool for predicting therapeutic efficacy. Adjustments between measure items, and differences between men and women and different age groups, are topics for further study.

\section{Acknowledgements}

This work was supported by the Grant in aid for Scientific Research (C) from the Japan Society for the Promotion of Science (grant numbers 22592451) and Grant-in-Aid for Research in Nagoya City University. The authors wish to thank sincerely all the patients who participated in this study.

\section{Conflict of Interest}

The authors have no financial conflicts of interest to disclose concerning the research. 


\section{References}

[1] The Japanese Society of Hypertension (2014) The Japanese Society of Hypertension Guidelines for the Management of Hypertension (JSH2014). Tokyo.

[2] World Health Organization (2013) WHO: A Global Brief On Hypertension: Silent Killer, Global Public Health Crisis. http://www.who.int/features/2013/japan_blood_pressure/en/index.html\#

[3] The Ministry of Health (2006) Labor and Welfare: The National Medical Expenditures in the Heisei 16 Fiscal Year. http://www.mhlw.go.jp/toukei/saikin/hw/k-iryohi/04/kekka6.html

[4] Mendis, S. and Salas, M. (2013) Hypertension, in World Health Organization, Adherence to Long-Term Therapies: Evidence for Action, 107-114. Geneva. http://www.who.int/chp/knowledge/publications/adherence full report.pdf

[5] Cohen, S.M. (2009) Concept Analysis of Adherence in the Context of Cardiovascular Risk Reduction. Nursing Forum, 44, 25-36. http://dx.doi.org/10.1111/j.1744-6198.2009.00124.x

[6] Bangalore, S., Kamalakkannan, G., Parkar, S. and Messerli, F.H. (2007) Fixed-Dose Combinations Improve Medication Compliance: A Meta-Analysis. American Journal of Medicine, 120, 713-719. http://dx.doi.org/10.1016/j.amjmed.2006.08.033

[7] Schroeder, K., Fahey, T. and Ebrahim, S. (2004) How Can We Improve Adherence to Blood Pressure-Lowering Medication in Ambulatory Care? Systematic Review of Randomized Controlled Trials. Archives of Internal Medicine, 164, 722-732. http://dx.doi.org/10.1001/archinte.164.7.722

[8] Collins, U.K. (1999) Collins Concise Dictionary. 4th Edition, HarperCollins Publishers, Glasgow, 16.

[9] Rajpura, J. and Nayak, R. (2014) Medication Adherence in a Sample of Elderly Suffering from Hypertension: Evaluating the Influence of Illness Perceptions, Treatment Beliefs, and Illness Burden. Journal of Managed Care Pharmacy, 19, 58-65.

[10] Abel, W.M. and Efird, J.T. (2013) The Association between Trust in Health Care Providers and Medication Adherence among Black Women with Hypertension. Front Public Health, 1, 66.

[11] Harmon, G., Lefante, J. and Krousel-Wood, M. (2006) Overcoming Barriers: The Role of Providers in Improving Patient Adherence to Antihypertensive Medications. Current Opinion in Cardiology, 21, 310-315. http://dx.doi.org/10.1097/01.hco.0000231400.10104.e2

[12] Gerbino, P.P. and Shoheiber, O. (2007) Adherence Patterns among Patients Treated with Fixed-Dose Combination versus Separate Antihypertensive Agents. American Journal of Health-System Pharmacy, 64, 1279-1283. http://dx.doi.org/10.2146/ajhp060434

[13] Lee, J.K., Grace, K.A. and Taylor, A.J. (2006) Effect of a Pharmacy Care Program on Medication Adherence and Persistence, Blood Pressure, and Low-Density Lipoprotein Cholesterol: A Randomized Controlled Trial. The Journal of the American Medical Association, 296, 2563-2571. http://dx.doi.org/10.1001/jama.296.21.joc60162

[14] Wee, L.E. and Koh, G.C. (2012) Individual and Neighborhood Social Factors of Hypertension Management in a Low-Socioeconomic Status Population: A Community-Based Case-Control Study in Singapore. Hypertension Research, 35, 295-303. http://dx.doi.org/10.1038/hr.2011.187

[15] Working Group on the Summit on Combination Therapy for CVD (2014) Combination Pharmacotherapy to Prevent Cardiovascular Disease: Present Status and Challenges. European Heart Journal, 35, 353-364. http://dx.doi.org/10.1093/eurheartj/eht407

[16] Morisky, D.E., Green, L.W. and Levine, D.M. (1986) Concurrent and Predictive Validity of a Self-Reported Measure of Medication Adherence. Medical Care, 24, 67-74. http://dx.doi.org/10.1097/00005650-198601000-00007

[17] Morisky, D.E., Ang, A., Krousel-Wood, M. and Ward, H.J. (2008) Predictive Validity of a Medication Adherence Measure in an Outpatient Setting. Journal of Clinical Hypertension, 10, 348-354.

http://dx.doi.org/10.1111/j.1751-7176.2008.07572.x 